\title{
Solubility of gases in polymers
}

\author{
Isaac C. Sanchez and Patrick A. Rodgers \\ Chemical Engineering Department and Center for Polymer Research, \\ The University of Texas at Austin, Austin, Texas 78712
}

\begin{abstract}
Solubility of gases in liquid polymers is treated theoretically using the lattice fluid model. Qualitatively, the model indicates that gas solubility should increase with molecular size as observed experimentally. In addition, the physical properties of the gas and polymer should dominate gas solubility with the gas-polymer interaction playing a secondary role. Using no adjustable parameters, gas solubilities can be quantitatively predicted for hydrocarbon and chlorinated hydrocarbon vapors in non-polar polymers. Good results are also obtained for polar gases (excluding alcohols) in polar polymers. Polar/non-polar combinations are not correlated as well, but the deviations are very systematic and suggest future directions for research.
\end{abstract}

\section{INTRODUCTION}

From a practical viewpoint, solubility of gases in polymers is an important consideration in removing volatiles from polymers, designing polymeric barrier materials for packaging applications, and developing membranes for gas separation. Although a complete understanding of gas solubility is lacking, excellent empirical correlations have been developed which quantitatively predict infinite dilution solubility of various organic vapors in several polymers (ref. 1-7). The logarithm of the solubility has been found to be a linear function of $\left(T_{c} / T\right)^{2}$ (ref. 1-3) where $T_{c}$ is the critical temperature of the gas. A somewhat better correlation is obtained when the log of the product of the solubility and the gas critical pressure is plotted against $\left(T_{\tau} / T\right)^{2}$ (ref. 4-7). However, the correlation is not universal in the sense that the constants $m$ and $b$ in the linear correlation, $m\left(T_{c} / T\right)^{2}+b$, depend on the specific polymer and on the chemical nature of the gas. An example of the latter is that aromatic and aliphatic hydrocarbons have different values of $b$ in poly(vinyl acetate) (ref. 3). The slope $m$ has also been correlated with the acentric factor which characterizes molecular size and shape (ref. 8). These empirical correlations imply that the gas-polymer interaction is of secondary importance in establishing solubility. The primary factor is the physical properties of the gas, as manifested in its critical properties, and the polymer.

In this paper we examine the solubility of gases in polymers as predicted by the lattice fluid (LF) model (ref. 9-12). The LF model is an equation of state model which takes into account the PVT properties of both gas and polymer. As will be seen, this model makes two qualitative predictions which are in excellent agreement with experiment. First, the gas-polymer interaction energy has only a second order effect on solubility as suggested by the aforementioned empirical correlations. Second, for a homologous series of gases, such as the normal alkanes, solubility should increase with molecular size as is observed. Quantitatively, it will be shown that the LF model can make excellent solubility predictions for non-polar gases in non-polar liquid polymers with no adjustable parameters. The temperature dependence of the solubility is also accurately given. For polar polymers and polar gases (alcohols excluded), good predictions are also obtained without adjustable parameters. However, most nonpolar/polar combinations of gas and polymer do not yield good results.

Much of the available experimental data on solubility of gases in liquid polymers comes from inverse gas chromatography (IGC). A fundamental quantity measured in the IGC experiment is the specific retention volume, $\mathrm{V}_{\mathrm{g}}^{0}$, of the probe gas. It is a direct measure of the solubility of the probe gas in the polymer. A theoretical relationship is derived to calculate $V_{g}^{0}$ from the LF model.

\section{THEORY}

In the IGC experiment the fundamental quantity that is measured is the net retention volume $V_{N}$ :

$$
V_{N} \equiv V_{R}-V_{0}=\left(t_{p}-t_{m}\right) V_{0} / t_{m}
$$

where $V_{R}$ is the volume of gas needed to elute the probe gas (retention volume), $V_{0}$ is the volume of gas needed to elute a non-interacting marker gas (void volume), $t_{p}$ is the elution time for the probe gas, and $t_{m}$ is the elution 
time for the marker. The stationary phase in the IGC experiment is a polymer above its glass transition or melting temperature. Under the right experimental conditions, equilibrium between the probe gas (component 1) and the liquid polymer (component 2) can be obtained; $V_{N}$ then approaches a constant value and is related to the thermodynamic partitioning of the probe between the gas and polymer phases (ref. 13)

$$
\mathrm{V}_{\mathrm{N}}=\mathrm{V}_{2}\left(\mathrm{c}_{1}^{\mathrm{liq}} / \mathrm{c}_{1}^{\mathrm{gas}}\right)
$$

where $\mathrm{V}_{2}$ is the volume of polymer, $c_{1}^{\text {liq }}$ is the concentration (mass/unit volume) of probe dissolved in the liquid polymer, and $c_{1}^{g a s}$ is the concentration of probe in the gas phase. Dividing both sides of eq. (2) by the polymer mass $\left(\mathrm{m}_{2}\right)$ yields the specific retention volume $\mathrm{V}_{\mathrm{g}}$ :

$$
\mathrm{V}_{\mathrm{g}} \equiv \mathrm{V}_{\mathrm{N}} / \mathrm{m}_{2}=\left(\mathrm{c}_{1}^{\mathrm{liq}} / \mathrm{c}_{1}^{\mathrm{gas}}\right) / \rho_{2}
$$

where $p_{2}$ is the mass density of the polymer. The amount of probe gas in the column is small and its partial pressure $\left(P_{1}\right)$ is very low so that to an excellent approximation it can be described by the ideal gas law:

$$
c_{1}^{\text {gas }}=P_{1} M_{1} / R T
$$

where $\mathrm{M}_{1}$ is the molecular weight of the probe gas. The amount of probe gas absorbed by the polymer is also very small so that we may use Henry's law to an excellent approximation to estimate $c_{1}^{\text {liq }}$ :

$$
P_{1}=k_{H} c_{1}^{\text {liq }}
$$

Substituting eqs. (4) and (5) into (3) yields

$$
V_{g}=k_{H}^{-1}\left(R T / M_{1} \rho_{2}\right)
$$

Note that $\mathrm{V}_{\mathrm{g}}$ does not depend on the partial pressure of the probe gas. In practice the amount of probe gas is extrapolated to zero to insure that the ideal gas law and Henry's law are obeyed (ref 14). Often $\mathrm{V}_{\mathrm{g}}$ is "corrected" to the standard temperature $T_{0}=273.15$ and the corrected specific retention volume $\mathrm{V}_{\mathrm{g}}^{0}$ is given by

$$
\mathrm{V}_{\mathrm{g}}^{0} \equiv \mathrm{V}_{\mathrm{g}}\left(\mathrm{T}_{0} / \mathrm{T}\right)=\mathrm{k}_{\mathrm{H}}^{-1}\left(\mathrm{RT}_{0} / \mathrm{M}_{1} \rho_{2}\right)
$$

$\mathrm{k}_{\mathrm{H}}^{-1}$ is the gas solubility per unit pressure and thus $\mathrm{V}_{\mathrm{g}}$ or $\mathrm{V}_{\mathrm{g}}^{0}$ is a direct measure of gas solubility in a polymer.

Obtaining a theoretical Henry's law constant begins by equating the chemical potential of the gas at temperature $\mathrm{T}$ and pressure $\mathrm{P}$ to the chemical potential of the gas absorbed in the polymer; i.e.,

$$
\mu_{1}^{0}(T, P, \text { free gas })=\mu_{1}(T, P, \text { absorbed gas }) .
$$

For the lattice fluid (LF) model the chemical potentials are given by (ref. 11):

and

$$
\mu_{1}^{0} / R T=r_{1}\left[\left(-\tilde{\rho}_{1}+\tilde{P}_{1} \tilde{v}_{1}\right) / \tilde{T}_{1}+\left(1-\tilde{\rho}_{1}\right) \ln \left(1-\tilde{\rho}_{1}\right) / \tilde{\rho}_{1}+\ln \tilde{\rho}_{1} / r_{1}\right]
$$

$$
\begin{aligned}
\mu_{1} / R T= & \ln \phi_{1}+\left(1+v_{1}^{*} / v_{2}^{*}\right) \phi_{2}+\tilde{\rho} v_{1}^{*} \chi \phi_{2}^{2} \\
& r_{1}\left[\left(-\tilde{\rho}+\tilde{P}_{1} \tilde{v}\right) / \tilde{T}_{1}+(1-\tilde{\rho}) \ln (1-\tilde{\rho}) / \tilde{\rho}+\ln \tilde{\rho} / r_{1}\right]
\end{aligned}
$$

where $\chi$ is the "bare" gas-polymer interaction parameter. The remaining undefined quantities are equation of state parameters and variables; the LF equation of state is

$$
\tilde{\rho}=1-\exp \left[-\left(\tilde{\rho}^{2}+\tilde{P}\right) / \tilde{T}-(1-1 / r) \tilde{p}\right]
$$

where $\tilde{\rho} \equiv \rho / \rho^{*}, \tilde{P} \equiv P / P^{*}$, and $\tilde{T} \equiv T / T^{*}$ are the reduced (dimensionless) mass density, pressure, and temperature, respectively. The mass density, pressure, and temperature are reduced by their respective equation of state parameters $\rho^{*}, P^{*}$, and $T^{*} ; r$ is a dimensionless size parameter proportional to the molecular weight (M):

$$
r=M\left(P^{*} / R^{*} \rho^{*}\right) \equiv P^{*} v^{*} / R^{*}
$$


$\mathrm{P}^{*}$ is the hypothetical cohesive energy density of the liquid at absolute zero temperature and $\mathrm{p}^{*}$ is the corresponding mass density; $\mathrm{T}^{*}$ does not have a clear physical significance (ref. 11). The close-packed or hard core molecular volume $\mathrm{v}^{*}$ is given by

$$
v^{*} \equiv M / \rho^{*}
$$

The $\phi_{i}$ in eq. (10) are close-packed volume fractions and are related to weight fractions $w_{i}$ by

$$
\phi_{i}=\frac{w_{i} / \rho_{i}^{*}}{\sum_{i} w_{i} / \rho_{i}^{*}}
$$

If the gas density $\rho_{1}$ is low so that $\tilde{\rho}_{1} \rightarrow 0$, then eq. (9) becomes

$$
\mu_{1}^{0} / \mathrm{RT}=\ln \tilde{\rho}_{1}-\mathrm{r}_{1}+1
$$

since $\mathrm{r}_{1} \tilde{\mathrm{P}}_{1} / \tilde{p}_{1} \tilde{\mathrm{T}}_{1}=\mathrm{PV} / \mathrm{RT} \rightarrow 1$ in this limit. Now $\mathrm{p}_{1}$ can be replaced by its ideal gas value, $\mathrm{PM} \mathrm{M}_{1} / \mathrm{RT}$, so that

$$
\mu_{1}^{0} / R T=\ln \left(P_{1} / \rho_{1}^{*} R T\right)-r_{1}+1 .
$$

In the dilute limit of gas absorption $\left(\phi_{1} \rightarrow w_{1} \rho_{2}^{*} / \rho_{1}^{*} \rightarrow 0\right)$ and high molecular weight $\left(M_{2}\right.$ and $\left.v_{2}^{*} \rightarrow \infty\right)$, eq. (10) becomes

$$
\mu_{1} / R T=\ln \left(w_{1} \rho_{2}^{*} / \rho_{1}^{*}\right)+1+\tilde{\rho}_{2} v_{1}^{*} \chi+r_{1}\left[-\tilde{\rho}_{2} / \tilde{T}_{1}+\left(1-\tilde{\rho}_{2}\right) \ln \left(1-\tilde{\rho}_{2}\right) / \tilde{\rho}_{2}+\ln \tilde{\rho}_{2} / r_{1}\right]
$$

where $\tilde{\rho}_{2}$ is the reduced density of the polymer liquid. The liquid PV term is very small compared to all others and has been ignored in eq.(16). Equating eqs.(15b) and (16) yields Henry's law [see eq. (5)] with $\mathrm{k}_{\mathrm{H}}^{-1}$ given by

$$
\mathrm{k}_{\mathrm{H}}^{-1}=\frac{\mathrm{M}_{1}}{\mathrm{RT}} \exp \left\{\mathrm{r}_{1}\left[\tilde{\rho}_{2} / \tilde{\mathrm{T}}_{1}-1-\left(1-\tilde{\rho}_{2}\right) \ln \left(1-\tilde{\rho}_{2}\right) / \tilde{\rho}_{2}\right]-\tilde{\rho}_{2} \mathrm{v}_{1}^{*} \chi\right\}
$$

which is a result that has been obtained before (ref 12,15). Substituting eq.(17) into (7) yields the desired relationship between the specific retention volume measured in an IGC experiment and the theoretical parameters of the LF model:

$$
\mathrm{V}_{\mathrm{g}}^{0}=\frac{\mathrm{T}_{0}}{\mathrm{~T} \rho_{2}} \exp \left\{\mathrm{r}_{1}\left[\tilde{\rho}_{2} / \tilde{T}_{1}-1-\left(1-\tilde{\rho}_{2}\right) \ln \left(1-\tilde{\rho}_{2}\right) / \tilde{\rho}_{2}\right]-\tilde{\rho}_{2} \mathrm{v}_{1}^{*} \chi\right\}
$$

or equivalently,

$$
\mathrm{V}_{\mathrm{g}}^{0}=\frac{\mathrm{T}_{0}}{\mathrm{~T} \rho_{2}} \exp \left\{\tilde{\rho}_{2} \frac{\mathrm{M}_{1}}{\rho_{1}^{*}}\left[\mathrm{P}_{1}^{*} / \mathrm{RT}-\chi\right]-\mathrm{r}_{1}\left[1+\left(1-\tilde{\rho}_{2}\right) \ln \left(1-\tilde{\rho}_{2}\right) / \tilde{\rho}_{2}\right]\right\} .
$$

$\mathrm{V}_{\mathrm{g}}^{0}$ has a simple interpretation: it represents the volume of probe gas, corrected to standard temperature $\left(\mathrm{T}_{0}=\right.$ $273.15 \mathrm{~K}$ ) and pressure $(1 \mathrm{~atm})$, that is absorbed by unit mass of polymer when the probe gas partial pressure is 1 atmosphere. To see this multiply Henry's law, eq.(5), by $\mathrm{RT}_{0} / \mathrm{P}_{0} \mathrm{M}_{1} \rho_{2}$ where $\mathrm{P}_{0}$ is some arbitrary reference pressure:

$$
\begin{aligned}
c_{1}^{\text {liq }}\left(R T_{0} / P_{0} M_{1} \rho_{2}\right)= & \begin{array}{l}
\text { volume of probe gas, corrected to } T_{0} \text { and } P_{0}, \text { absorbed per unit } \\
\text { mass of polymer at a partial pressure of } P_{1} .
\end{array} \\
= & k_{H}^{-1}\left(R T_{0} / M_{1} P_{2}\right)\left(P_{1} / P_{0}\right)=V_{g}^{0}\left(P_{1} / P_{0}\right)
\end{aligned}
$$

where the last equality follows from eq.(7). Thus, if the probe gas had a partial pressure $\left(P_{1}\right)$ of $1 \mathrm{~atm}$, the volume of gas, corrected to STP conditions, absorbed by unit mass of polymer equals $\mathrm{V}_{\mathrm{g}}^{0}$. This interpretation only depends on the validity of the ideal gas law and Henry's law up to pressures of $1 \mathrm{~atm}$; if their validity only holds up to a pressure of $P_{0}$. then $V_{g}^{0}$ equals the volume of probe gas, corrected to standard temperature $\left(T_{0}=273.15 \mathrm{~K}\right)$ and reference pressure $P_{0}$, that is absorbed by unit mass of polymer when the probe gas partial pressure is $P_{0}$. 


\section{COMPARISON WITH EXPERIMENT}

A pure component is completely characterized by the three equation of state parameters; these parameters have been tabulated for many small molecules (ref. 9) as well as for several polymers (ref. 16). For small molecules, the parameters can be determined from a latent heat of vaporization, a vapor pressure and a liquid density (ref. 17); for polymers, a density, thermal expansion coefficient, and isothermal compressibility are required (ref. 16) or a nonlinear regression of PVT data with the theoretical equation of state can be used. In Tables 1 and 2, equation of state parameters are tabulated for the probe gases (40) and polymers (5) considered herein.

Table 1. Characteristic Parameters for 40 Gas Probes

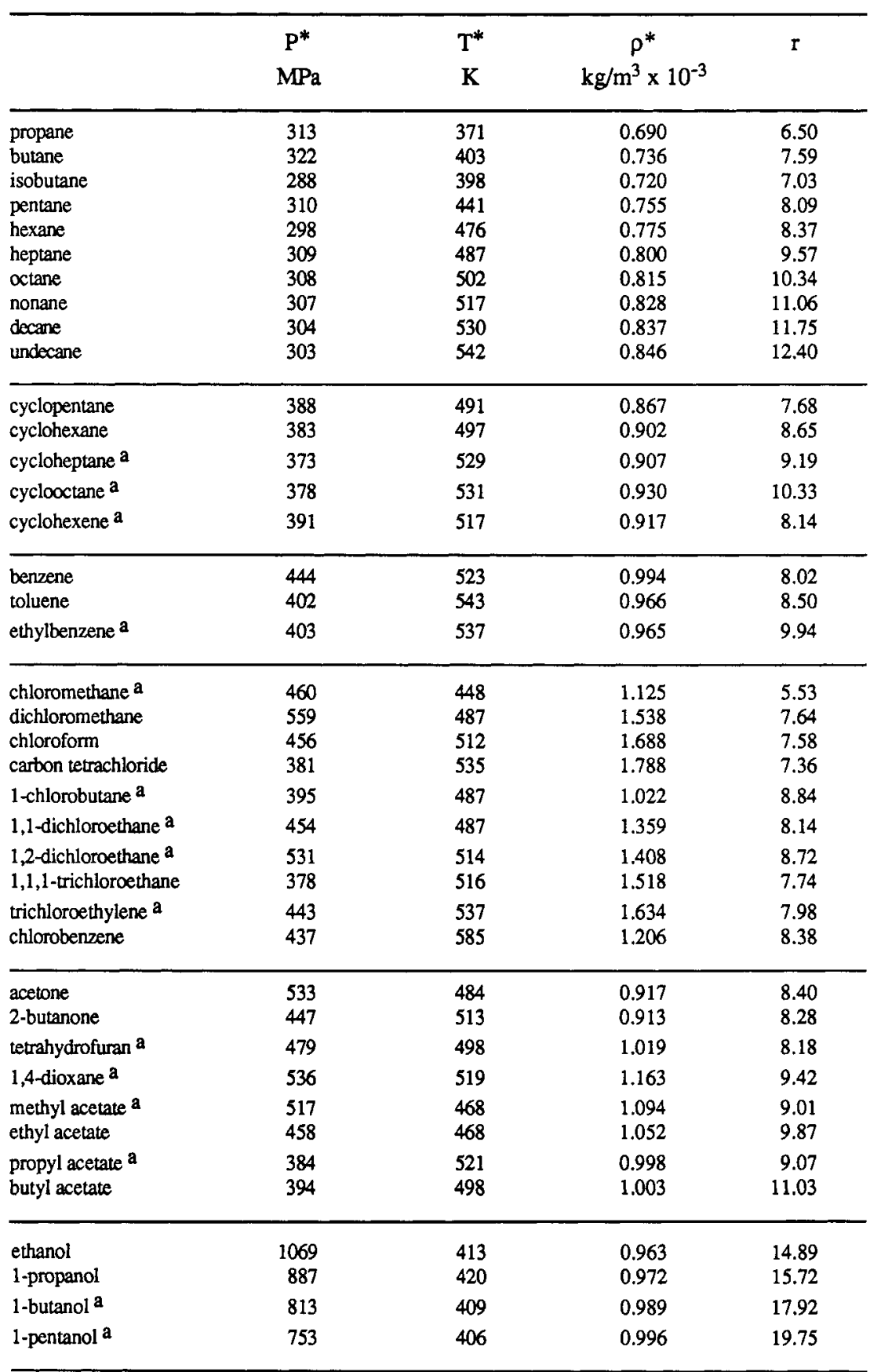

a Parameters for these compounds determined from a latent heat of vaporization, vapor pressure, and liquid density, all at a given temperature, as described in Appendix $\mathrm{A}$ in ref [17]. Parameters for the remaining compounds taken from ref [9]. 
Table 2. Characteristic Parameters for 5 Polymers

\begin{tabular}{lccccr}
\hline & $\begin{array}{c}\mathrm{P}^{*}, \\
\mathrm{MPa}\end{array}$ & $\begin{array}{c}\mathrm{T}^{*}, \\
\mathrm{~K}\end{array}$ & $\begin{array}{c}\rho^{*}, \\
\mathrm{~kg} / \mathrm{m}^{3} \times 10^{-3}\end{array}$ & $\begin{array}{c}\text { temp. } \\
\text { range, } \mathrm{K}\end{array}$ & $\begin{array}{c}\operatorname{max.} \mathrm{P} \\
\mathrm{MPa}\end{array}$ \\
\hline polystyrene $^{\mathrm{a}}$ & 357 & 735 & 1.105 & $388-468$ & 200 \\
poly(vinyl acetate) $^{\mathrm{a}}$ & 509 & 590 & 1.283 & $308-373$ & 80 \\
poly(methyl acrylate) $^{\mathrm{b}}$ & 514 & 604 & 1.263 & $313-493$ & 200 \\
polypropylene (iso) $^{\mathrm{c}}$ & 281 & 771 & 0.852 & $473-553$ & 20 \\
poly(1-butene) $^{\mathrm{c}}$ & 318 & 730 & 0.880 & $423-503$ & 20 \\
\hline
\end{tabular}

a Parameters taken from ref [16].

b Parameters determined from PVT data provided by DJ. Walsh and as described in ref.[24].

c Parameters given in ref [25]. PVT data on atactic-polypropylene are not available.

In eq.(18) $\tilde{p}_{2}$ is the reduced mass density of the polymer which is calculated from the equation of state, eq.(11). The only unknown parameter in equation (18) is $\chi$ and it is given by (ref. 11)

$$
\mathrm{RT} \chi \equiv \Delta \mathrm{P}^{*} \equiv \mathrm{P}_{1}^{*}+\mathrm{P}_{2}^{*}-2 \xi\left(\mathrm{P}_{1}^{*} \mathrm{P}_{2}^{*}\right)^{1 / 2}
$$

where $\xi$ is a dimensionless parameter near unity; if both the gas and polymer are non-polar, it is expected that $\xi=1$ (geometric mean approximation) so that

$$
\Delta \mathrm{P}^{*}=\left[\left(\mathrm{P}_{1}^{*}\right)^{1 / 2}-\left(\mathrm{P}_{2}^{*}\right)^{1 / 2}\right]^{2}
$$

Since $P_{i}^{*}$ is the cohesive energy density of component $i$ at zero absolute temperature, the above geometric approximation is analogous to the well-known approximation made for "regular solutions" at non-zero temperatures (ref. 18). In this approximation the heat of mixing at zero temperature would always be positive, but at non-zero temperatures, equation of state contributions (through volume contraction on mixing) can cause the heat of mixing to become negative.

If we invoke the geometric mean approximation, eq. (21), the solubility, or equivalently $\mathrm{V}_{\mathrm{g}}^{0}$, is completely determined by the gas and polymer pure component parameters listed in Tables I and II. In Fig. 1 this approximation is used to predict $V_{g}^{0}$ for 6 non-polar organics in polystyrene at $423 \mathrm{~K}, 448 \mathrm{~K}$, and $473 \mathrm{~K}$. In the remaining 4 figures, this approximation is tested for a variety of organics at $373 \mathrm{~K}$ in 2 non-polar polymers, poly(1butene) and atactic-polypropylene and 2 polar polymers, poly(vinyl acetate) and poly(methyl acrylate).

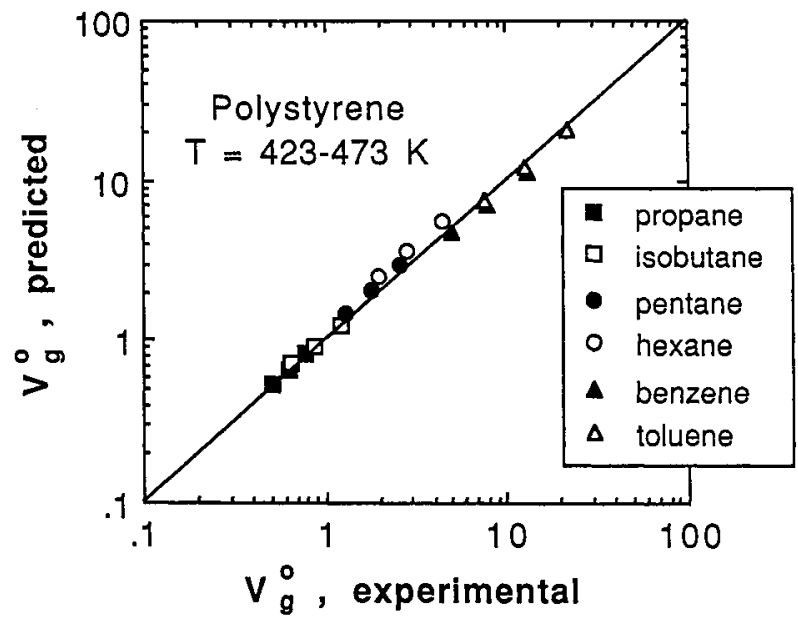

Fig. 1. Comparison of calculated [eqs. $18 \& 21$ ] and experimental values of IGC specific retention volumes (solubilities) for the indicated hydrocarbons in polystyrene @ 423,448, and $473 \mathrm{~K}$. Experimental data from ref. [2]. Retention volumes in units of $\mathrm{cm}^{3}$ of gas corrected to $273 \mathrm{~K}$ per gram of polymer. Largest percentage error $31 \%$ for hexane @ $448 \mathrm{~K}$. Average percentage error $11 \%$. 


\section{DISCUSSION}

Inspection of eqs. (17) and (18) immediately indicates that molecular size of the gas is an important parameter in determining solubility. By expanding the logarithm term it is easy to show that the sum of terms within the square braces in eqs. (17) and (18) is always positive for values of $\tilde{\mathrm{T}}_{1}<3 / 2$. This includes all temperatures of practical interest; from the $T^{*}$ values in Table $\mathrm{I}$, we see that $\tilde{T}_{1}<3 / 2$ corresponds to temperatures less than $700 \mathrm{~K}$, which is far above the thermal decomposition temperature of most polymers. Notice that this positive term is scaled by the the size parameter $r_{1}$ which is proportional to the molecular weight of the gas [ see eq. (12)]. Thus, the LF model qualitatively predicts that solubility should increase with increasing chain length for a homologous series of gases such as the alkanes. This is illustrated in Figs. 2-5 for the alkanes; propane is always much less soluble than undecane. The same behavior is also observed in the cyclic hydrocarbons. This is not a small effect. The ratio of solubilities between undecane and propane is typically about 500 . The solubility ratio between cyclooctane and cyclopentane falls between 10 and 15 . A virtue of the LF model, say compared to the Flory equation of state model (ref. 19-22), is that it is able to make explicit predictions about how molecular size affects properties. For example, the LF model correctly predicts that for a homologous series of liquids of increasinging size: liquid density increases, critical temperature increases, critical pressure decreases, liquid surface tension increases, and liquid surface entropy decreases (ref. 17, 23). In several cases these predictions are also quantitative.

Notice that the size term only depends on the pure component parameters of the gas and polymer and its size is typically an order of magnitude larger than the interaction term when $\chi$ is approximated by eq. (21). As mentioned in the Introduction, this indicates that the gas-polymer interaction energy plays a secondary role in determining gas

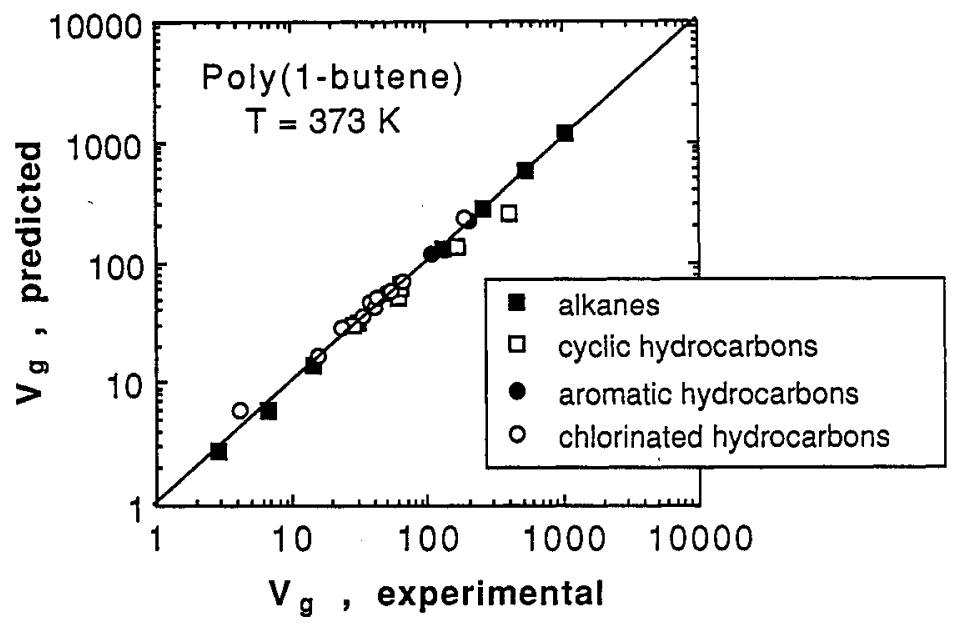

Fig. 2. Comparison of calculated [eqs. $18 \& 21$ ] and experimental values of IGC specific retention volumes for 27 hydrocarbons in poly(1-butene) @ $373 \mathrm{~K}$. Experimental data (uncorrected) from ref. [26]. Largest percentage error 50\% for methyl chloride. Average percentage error $13 \%$.

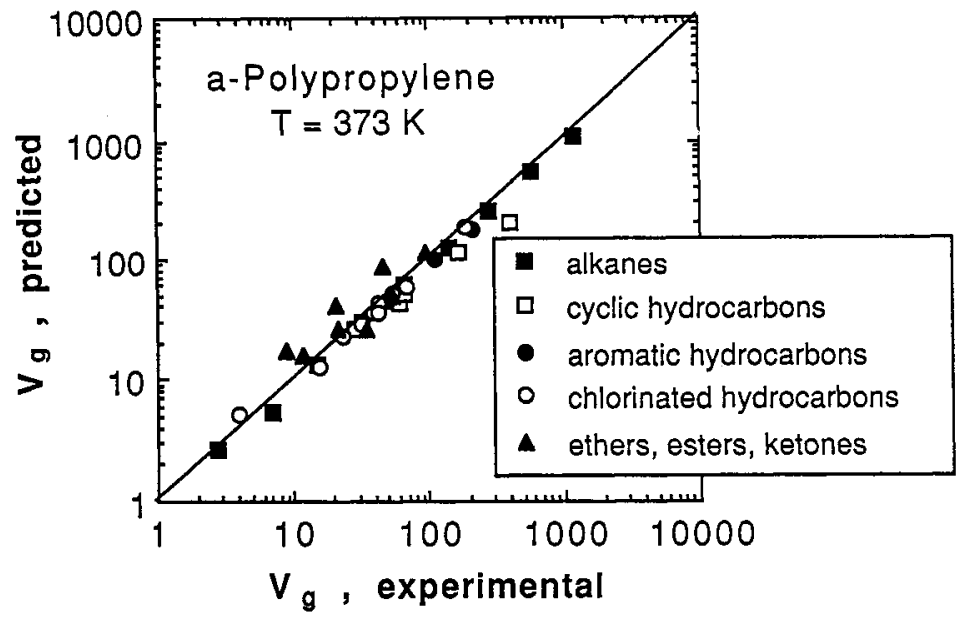

Fig. 3. Comparison of calculated [eq. 18] and experimental values of IGC specific retention volumes for 27 hydrocarbons and 8 oxygen containing organics in atactic-polypropylene @ $373 \mathrm{~K}$. Experimental data (uncorrected) from ref. [26]. Notice that the oxygen containing organics are systematically overestimated. Average percentage error for the 27 hydrocarbons $13 \%$; average error for the 8 oxygen containing organics $50 \%$. 


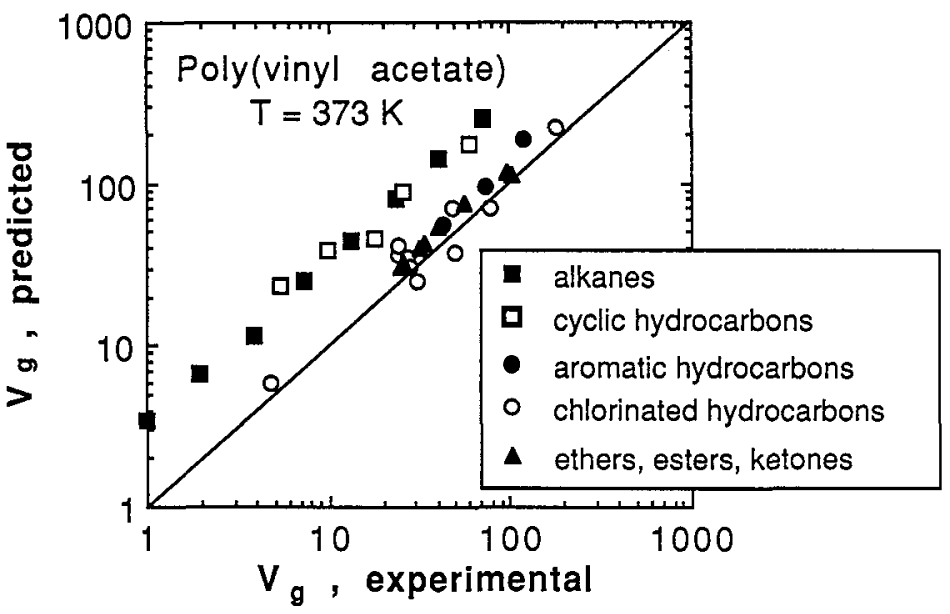

Fig. 4. Comparison of calculated [eqs. $18 \& 21$ ] and experimental values of IGC specific retention volumes for 27 hydrocarbons and 8 oxygen containing organics in poly(vinyl acetate) @ $373 \mathrm{~K}$. Experimental data (uncorrected) from ref. [27]. Notice that the aliphatic hydrocarbons (14) are systematically overestimated. Polar gases (18) and aromatic hydrocarbons (3) are reasonably correlated with an average percentage error of $30 \%$.

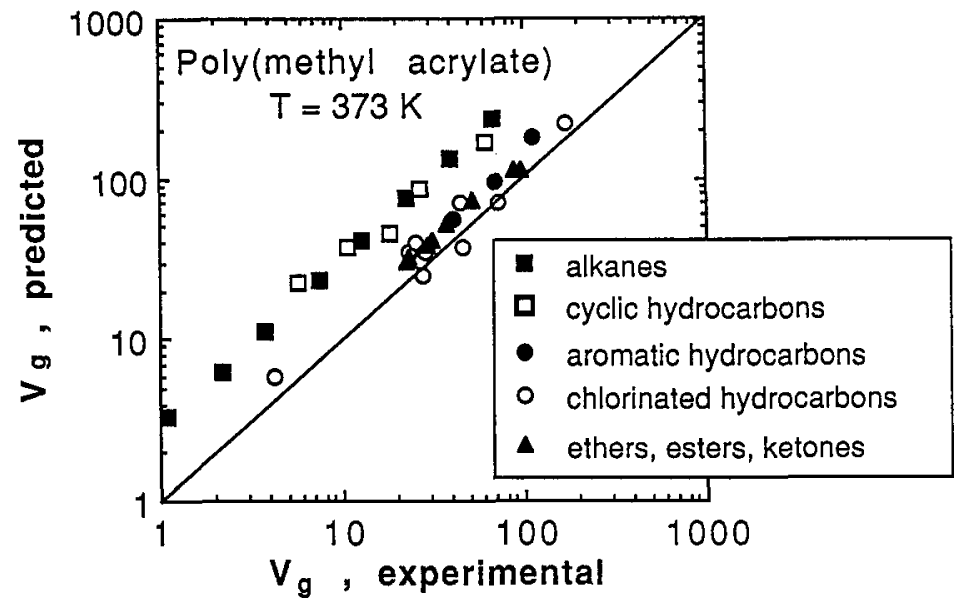

Fig. 5. Comparison of calculated [eqs. $18 \& 21$ ] and experimental values of IGC specific retention volumes for 27 hydrocarbons and 8 oxygen containing organics in poly(methyl acrylate) @ $373 \mathrm{~K}$. Experimental data (uncorrected) from ref. [27]. Notice that the aliphatic hydrocarbons (14) are systematically overestimated. Polar gases (18) and aromatic hydrocarbons (3) are reasonably correlated with an average percentage error of $34 \%$.

solubility; pure component properties dominate. However, the interaction term cannot be ignored. The systematic deviations that we have observed, some of which are illustrated in Figs. 3-5, are related to inaccurate estimates of the interaction energy by the geometric mean approximation.

A third qualitative prediction made by the LF model, also associated with the dominance of the size term, is that the temperature dependence of solubility increases as molecular size increases. Solubility decreases with increasing temperature and this effect will be more pronounced for large gas molecules. This effect can be observed qualitatively in Fig. 1; note that propane has a weaker dependence on temperature than the larger toluene. The temperature dependence of gas solubility predicted by the LF model has been more fully explored by Panayiotou (ref. 15)

For the 3 non-polar polymers shown in Figs. 1-3, we see an excellent correlation between experimental and calculated solubilities [using eq. 21)] for non-polar gases. For poly(1-butene) and atactic-polypropylene we also see, somewhat surprisingly, that the 10 chlorinated hydrocarbons listed in Table 1 are also correlated well. However, the solubilities of oxygen containing organics (ethers, esters, and ketones) in these 2 non-polar polymers are consistently overestimated; this is illustrated for atactic-polypropylene in Fig. 3. For associated liquids such as alcohols, the geometric mean approximation consistently and significantly underestimates solubilities in both nonpolar and polar polymers (not shown in any figure).

For the 2 polar polymers shown in Figs. 4 and 5, we see that the solubilities of non-aromatic hydrocarbons are grossly and systematically overestimated. Polar and aromatic gases are correlated reasonably well. The systematic deviations from the geometric mean are encouraging because it suggests that methods can be developed to estimate the interaction term based on the chemical structure of the gas and polymer. 


\section{Acknowledgements}

The authors would like to thank Prof. Petr Munk and his research group for valuable discussions and for providing access to their IGC data prior to publication (ref. 26 and 27). This research was supported in part by the National Science Foundation and the Air Force Office of Scientific Research.

\section{REFERENCES}

1. S.A. Stem, J.T. Mullhaupt and P.J. Gareis, AIChE J. 15, 64-73 (1969).

2. L.I. Stiel and D.F. Harnish, AIChE J, 22, 117-121 (1976).

3. H.-S. Tseng, D.R. Lloyd and T.C. Ward, Polym. Comm. 25, 262-264 (1984).

4. H.-S. Tseng, P.-C. Wong, T.C. Ward, J.W. Barlow and D.R. Lloyd, Polym. Mater. Sci. Eng, 51, 229-234 (1984).

5. H.-S. Tseng, D.R. Lloyd and T.C. Ward, J. Appl. Polym. Sci, 30, 307-315 (1985).

6. H.-S. Tseng, D.R. Lloyd and T.C. Ward, J. Appl. Polym. Sci 30 , 1815-1826 (1985).

7. H.-S. Tseng, P.-C. Wong, D.R. Lloyd and J.W. Barlow, Polym. Eng. Sci. 27, 1141-1147 (1987).

8. L.I. Stiel, D.-K. Chang, H.-H. Chu and C.-D. Han, J. Appl. Polym. Sci. 30, 1145-1165 (1985).

9. I.C. Sanchez and R.H. Lacombe, J. Phys, Chem, 80, 2352-2362 (1976).

10. R.H. Lacombe and I.C. Sanchez, J. Phys, Chem, 80, 2568-2580 (1976).

11. I.C. Sanchez, "Polymer Phase Separation" pp. 1-18 in Encyclopedia of Physical Science and Technology, Vol 11, (R. A. Meyers, ed.) Academic Press, New York (1987).

12. I.C. Sanchez, Polymer 30, 471-475 (1989).

13. P. Munk, Introduction to Macromolecular Science, p. 473-475, Wiley, New York (1989).

14. P. Munk, Z.Y. Al-Saigh and T.W. Card, Macromolecules 18, 2196-2201 (1985).

15. C.G. Panayiotou, Makromol. Chem, 187, 2867-2882 (1986).

16. I.C. Sanchez and R.H Lacombe, J. Polym. Sci. Polvm. Lett. Ed, 15, $71-75$ (1977).

17. I.C. Sanchez and R.H Lacombe, Mactomolecules 11, 1145-1156 (1978).

18. J.H. Hildebrand, J.M. Prausnitz and R.L. Scott, Regular and Related Solutions, pp. 82-91, Van Nostrand Reinhold, New York (1970).

19. P.J. Flory, R.A. Orwoll and A.J. Vrij, J. Am. Chem. Soc. 86, 3515-3520 (1964).

20. P.J. Flory, J. Am, Chem. Soc. 87, 1833-1838 (1965).

21. B.E. Eichinger and P.J. Flory, Trans. Faraday Soc. 64, 2035-2052 (1968).

22. P.J. Flory, Discuss, Faraday Soc 49, 7-29 (1970).

23. I.C. Sanchez and C.I. Poser, "Surface Thermodynamics of Liquid Polymers: Theory" pp. 173-182 in Physicochemical Aspects of Polymer Surfaces, Vol. 1 (K.L. Mittel, ed.), Plenum Press, New York (1983).

24. G.T. Dee and D.J. Walsh, Macromolecules 21, 811-815 (1988).

25. M.T. Pottiger and R.L. Laurence, J. Polym. Sci. Polym. Phys, Ed. 22, 903-907 (1984).

26. Q. Du, P. Hattam and P. Munk, "Inverse Gas Chromatography 7. Polymer-Solvent Interactions of Hydrocarbon Polymers", J. Chem. Eng. Data (in press).

27. P. Munk, P. Hattam,Q. Du, and A.-A. A. Abdel-Azim "Determination of Polymer-Solvent Interaction Coefficients by Inverse Gas Chromatography", J. Applied Polvmer Science (submitted). 